\title{
Paradoxical worsening of lipid metabolism after successful treatment of primary aldosteronism
}

Adolf, Christian ${ }^{1}$, Asbach, Evelyn${ }^{1}$, Dietz, Anna Stephanie ${ }^{1}$, Lang, Katharina ${ }^{4}$, Hahner, Stefanie ${ }^{4}$, Quinkler Marcus $^{5}$, Rump Lars Christian $^{6}$, Bidlingmaier, Martin ${ }^{1}$, Treitl, Marcus ${ }^{2}$, Ladurner, Roland ${ }^{3}$, Beuschlein, Felix ${ }^{1}$, Reincke, Martin ${ }^{1}$

\section{Context:}

Primary aldosteronism (PA) describes the most frequent cause of secondary arterial hypertension. Recently, deterioration of lipid metabolism after adrenalectomy (ADX) for aldosterone-producing adenoma (APA) has been described.

\section{Objective:}

We analysed longitudinal changes in lipid profiles in a large prospective cohort of PA patients. Data of 215 consecutive PA patients with APA $(n=144)$ or bilateral idiopathic adrenal hyperplasia (IHA, $\mathrm{n}=71$ ) were extracted from the database of the German Conn's Registry. Patients were investigated before and one year after successful treatment by ADX or by mineralocorticoid receptor antagonists (MRA).

\section{Methods:}

Glomerular filtration rate (GFR), fasting plasma glucose and components of lipid metabolism including triglycerides (TG), total cholesterol (TC), low-density lipoprotein cholesterol (LDL-C) and highdensity lipoprotein cholesterol (HDL-C) were determined at 8.00 after a 12-hour fasting period.

\section{Figure 1: Role of CKD in Cholesterol Metabolism}

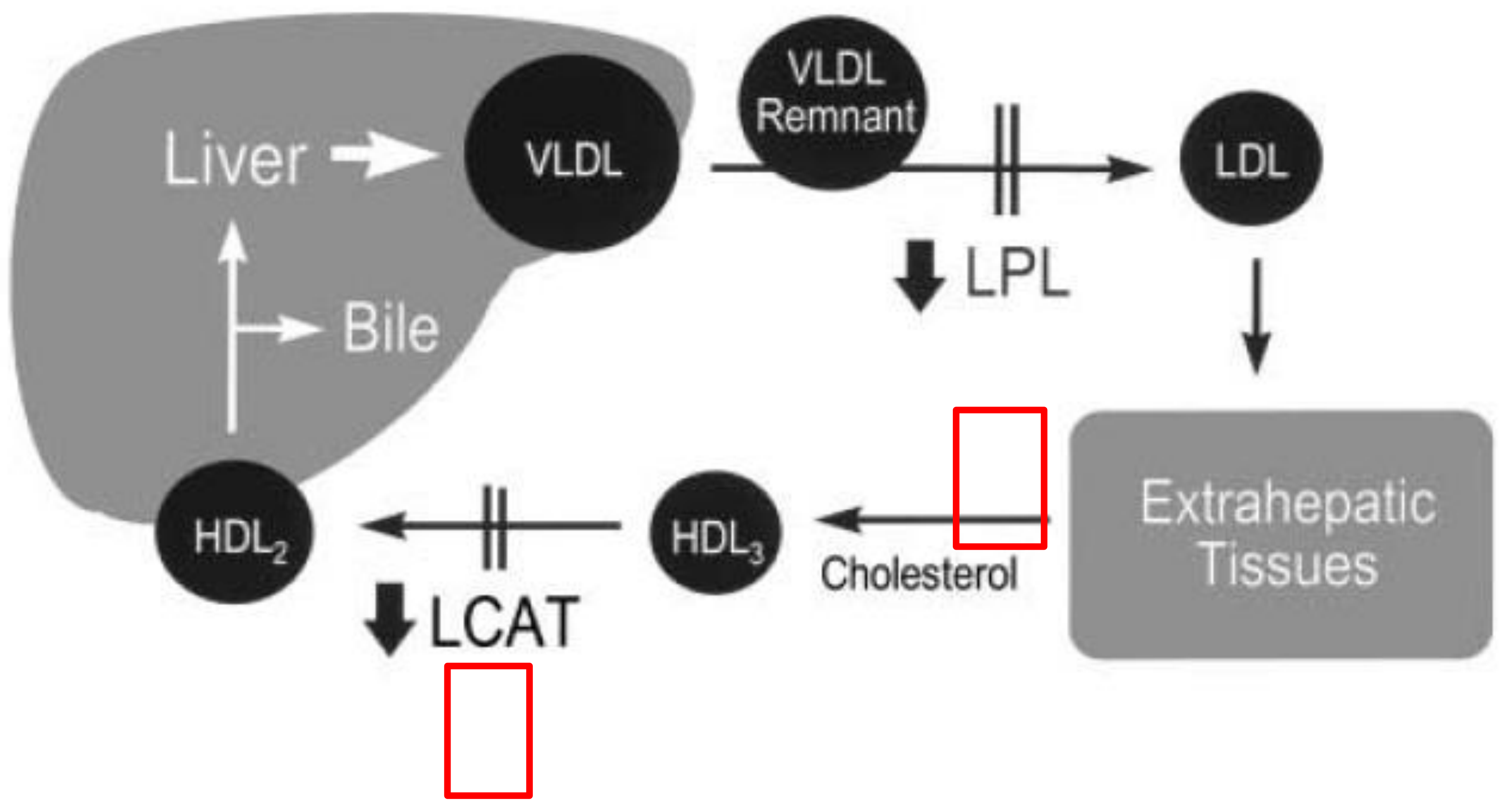

Abbreviations: LCAT, lecithin-cholesterol acyltransferase; LPL, lipoprotein lipase CKD, chronic kidney disease

Figure 3: Metabolic variables of PA patients before and one year after treatment

\begin{tabular}{|l|l|l|l|}
\hline Subtype & APA & APA & $p$ \\
\hline$n$ & 144 & 144 & -- \\
\hline Time of Assessment & Before ADX & One year after ADX & -- \\
\hline Serum Potassium & $3.7[3.2 ; 4.1]$ & $4.2[4.0 ; 4.5]$ & $0.00^{*}$ \\
\hline BMl [kg/m²] & $27.9[24.7 ; 32.2]$ & $28.0[24.6 ; 32.0]$ & 0.11 \\
\hline Fasting Plasma Glucose [mg/dl] & $99[90 ; 109]$ & $95[88 ; 104]$ & $0.00^{*}$ \\
\hline HDL-Cholesterol [mg/dl] & $53[45 ; 76]$ & $52[44 ; 65]$ & $0.04^{*}$ \\
\hline LDL-Cholesterol [mg/dl] & $122[95 ; 144]$ & $121[96 ; 141]$ & 0.39 \\
\hline Total Cholesterol [mg/dl] & $200[175 ; 224]$ & $200[175 ; 224]$ & 0.24 \\
\hline Triglycerides [mg/dl] & $104[69 ; 148]$ & $111[78 ; 167]$ & $0.00^{*}$
\end{tabular}

Results:

One year after initiation of treatment mean serum potassium levels and blood pressure normalized in the patients. HDL-C and TG developed inversely with decreasing HDL-C levels in patients with APA $(p=.046)$ and IHA $(p=.004)$ and increasing TG levels (APA $p=.000 ;$ IHA $p=.020)$. BMl remained unchanged and fasting plasma glucose improved in patients with APA $(p=.004)$. Furthermore, there was a significant decrease of GFR in both subgroups at follow-up $(p=.000)$. Changes in HDL-C and TG correlated with decrease in GFR in multivariate analysis $(p=.024)$.

\section{Conclusion:}

Treatment of PA is associated with a deterioration of lipid parameters despite stable BMI and improved fasting plasma glucose and blood pressure. This paradoxical effect can be explained by renal dysfunction following ADX or MRA-therapy.

\section{Figure 2: Lipid- and GFR changes following treatment}

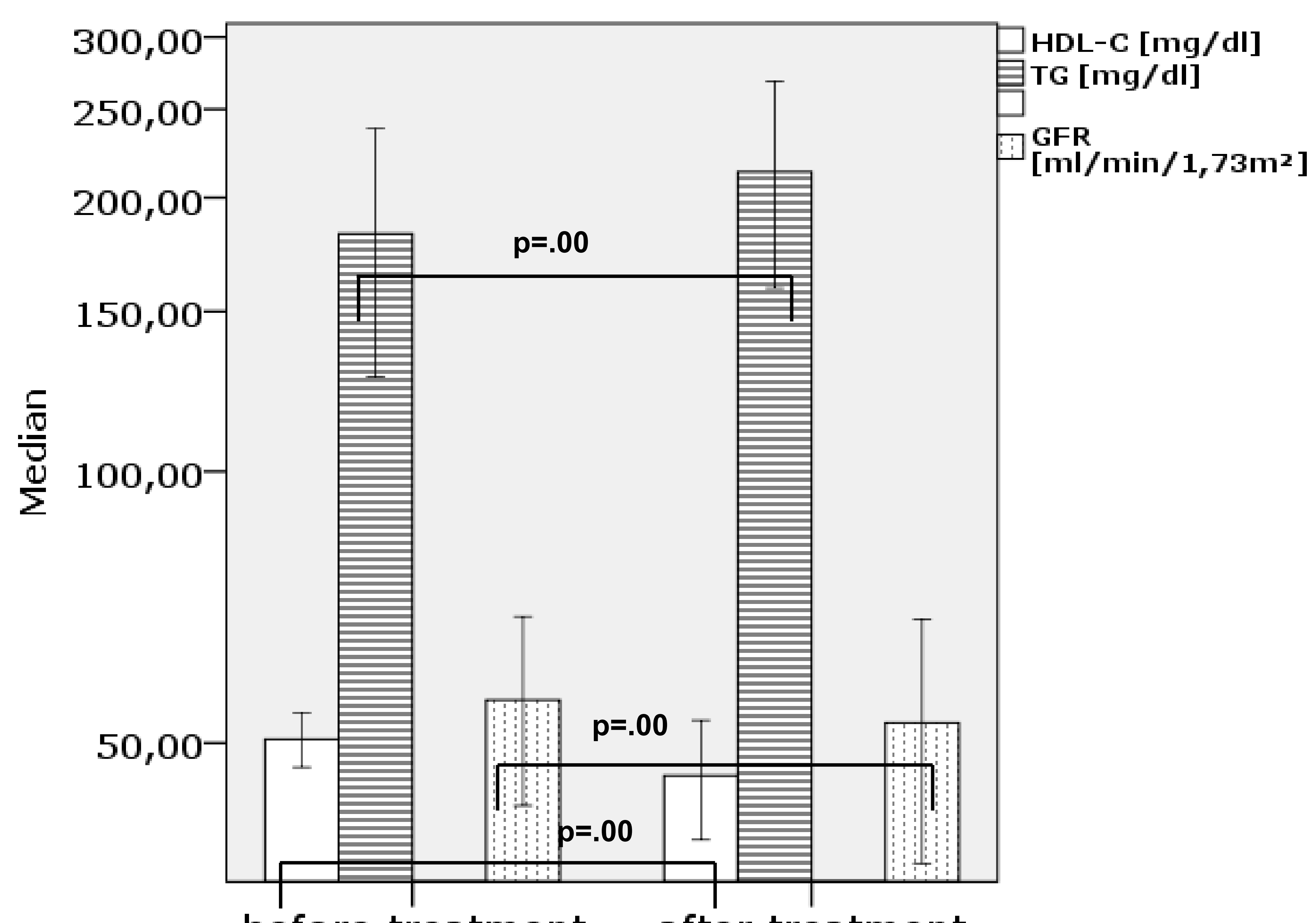

before treatment after treatment

time of assessment

\begin{tabular}{|l|ll}
\hline IHA & IHA & $\mathbf{p}$ \\
\hline 71 & 71 & - \\
Before MRA & One year after MRA & -- \\
\hline $3.7[3.3 ; 3.9]$ & $4.1[3.0 ; 4.4]$ & $0.00^{*}$ \\
$28.7[25.8 ; 31.0]$ & $29.0[25.5 ; 32.4]$ & 0.23 \\
$100[90 ; 120]$ & $102[94 ; 111]$ & 0.81 \\
\hline $52[42 ; 66]$ & $48[39 ; 62]$ & $0.00^{*}$ \\
$120[99 ; 143]$ & $116[101 ; 134]$ & 0.68 \\
$193[171 ; 216]$ & $192[171 ; 212]$ & 0.91 \\
$111[82 ; 150]$ & $129[85 ; 195]$ & $0.02^{*}$ \\
\hline
\end{tabular}

Medizinische Klinik und Poliklinik IV, Klinikum der Ludwig-Maximilians-Universität, München

2 Institut für Klinische Radiologie, Klinikum der Ludwig-Maximilians-Universität, München

3 Klinik für Viszeral- und Endokrine Chirurgie, Klinikum der Ludwig-Maximilians-Universität, München

4 Medizinische Klinik I, Julius-Maximilians-Universität, Würzburg

5 Endokrinologiepraxis in Charlottenburg, Berlin 